\title{
Effect of Parity on Pregnancy Outcome in Women with Type-1 Diabetes - A Swedish Population-based Study
}

\section{Martina Persson ${ }^{1,2 *}$ and Dharmintra Pasupathy ${ }^{1}$}

${ }^{1}$ Women's Health Academic Centre, King's Health Partners, King's College London, Guy's and St Thomas' NHS Foundation Trust, London, UK ${ }^{2}$ Clinical Epidemiology Unit, Department of Medicine Solna, Karolinska University Hospital, Karolinska Institutet, Stockholm, Sweden

\begin{abstract}
Aim: Women without diabetes are at increased risk of pregnancy complications in their first pregnancy. The aim of this study was to investigate if the risk of adverse pregnancy outcomes also differs with parity in women with type 1 diabetes (T1DM).

Methods: Population based prospective cohort study of all singleton pregnancies to women with T1DM ( $\mathrm{n}=4092)$ in Sweden between 1998 and 2007. Data was derived from the Swedish Medical Birth Registry. Outcomes of interest were preeclampsia $(\mathrm{PE})$, gestational hypertension $(\mathrm{GH})$, major malformation, perinatal mortality (PMR), preterm delivery, large for gestational age (LGA), small for gestational age (SGA) and neonatal death. Logistic regression analysis was used to obtain odds ratios (OR) for adverse outcomes in relation to parity. Interaction tests were performed to compare the association of interest in non-diabetic pregnancies.

Results: The risk of GH (adjusted OR $0.6395 \% \mathrm{Cl}$ [0.37-1.05]), major malformation (1.25 [0.88-1.77]), preterm delivery $(0.83[0.70-1.00])$ and neonatal death $(5.34$ [0.57-49.8]) did not differ significantly with parity in women with T1DM. Multiparous women with T1DM had lower risk of PE (0.35 [0.28-0.43]), SGA (0.44 [0.29-0.68]), PMR (0.22 $[0.10-0.51])$ and significantly increased odds of LGA $(1.96(1.71-2.25)$. In women without diabetes $(n=905565)$ the risk of all outcomes except LGA and neonatal death were significantly lower in multiparous women ( $p$ value for interaction < 0.05).

Conclusion: Multiparity in women with and without T1DM is associated with reduced risks for most adverse outcomes except LGA. Within the T1DM cohort, the risk for major malformations was slightly increased in offspring of multiparous women.
\end{abstract}

\section{Keywords: Diabetes; Pregnancy;Gestational; Women}

\section{Introduction}

It is well recognized that pregnancy in type 1 diabetes is associated with increased risks of adverse pregnancy outcomes, including preeclampsia, major malformations, perinatal mortality, birth trauma, preterm delivery and neonatal morbidities [1-4]. In women without type 1 diabetes, obstetric complications are more common in the first pregnancies (primiparous) compared with subsequent pregnancies and births (multiparas). These complications include preeclampsia, gestational hypertension, stillbirth, perinatal mortality, fetal growth restriction, preterm birth, instrumental delivery and delivery by cesarean section [5-12]. It has been proposed that a contributing factor to the increased risk in primiparous women could be a higher prevalence of maternal risk factors in this group, including lower maternal age, lower pre-pregnancy BMI, insufficient pregnancy weight gain, hypertensive disorders of pregnancy, poor education, noncohabiting, smoking and socioeconomic stress [13-15]. Primiparity may also convey an independent effect on the risk of preeclampsia with associated complications via immunological mechanisms unique to the first pregnancy [16]. The higher risk for complications in primiparas may also partly be attributed to the fact that fecundity issues may be more common in these women and also that higher-risk women are less likely to have subsequent pregnancies. However, this selection can only partly explain the observed reduced risk of complications with increasing parity [17].

In women with T1DM, maternal hyperglycemia is an important risk factor for almost all obstetric and perinatal complications [1820]. Other risk factors are high maternal BMI [21] and diabetic microangiopathy, in particular nephropathy [22]. However, it is unclear how parity affects the risk of perinatal complications in women with type 1 diabetes. This information is important in order to provide adequate pre-pregnancy counseling. The primary aim with the present study was to investigate if the risk of adverse pregnancy outcome differs between primiparous and multiparous women with type 1 diabetes. We hypothesized that the effect of parity on the risk for complications in pregnancies with type 1 diabetes would not be different from that in pregnancies without maternal diabetes.

\section{Source of Patient's Data}

This is a prospective nationwide cohort study based on data from the Swedish medical birth registry (MBR) from 1998-2007. The Swedish Medical Birth Register includes information on more than $98 \%$ of births in Sweden from 1973 [23]. Women with T1DM were identified based on ICD 10 code O 24.0 and only singleton pregnancies were included. Women without a diagnosis of diabetes (T1DM, T2DM or gestational diabetes) served as the reference group. The MBR provides

*Corresponding author: Martina Persson, Clinical Epidemiology Unit, Department of Medicine Solna, Karolinska University Hospital, Karolinska Institutet, Stockholm Sweden, Tel: +46 -8-73 980 5959; E-mail: Martina.Persson@ki.se

Received February 19, 2015; Accepted April 20, 2015; Published April 24, 2015

Citation: Persson M, Pasupathy D (2015) Effect of Parity on Pregnancy Outcome in Women with Type-1 Diabetes - A Swedish Population-based Study. J Diabetes Metab 6: 534. doi:10.4172/2155-6156.1000534

Copyright: @ 2015 Persson M, et al. This is an open-access article distributed under the terms of the Creative Commons Attribution License, which permits unrestricted use, distribution, and reproduction in any medium, provided the original author and source are credited. 
prospectively collected data on maternal demography, maternal and infant anthropometry and diagnoses. The registry also contains data on the mother's previous medical and obstetric history. The information is filled in by the midwife at the antenatal care and at delivery on a standardized form. All diagnoses are provided by a physician at discharge from hospital. Until 2008, the MBR only collected data on fetal deaths occurring from the $28^{\text {th }}$ week. No information on earlier fetal losses or induced abortions is available. In the MBR and for the purposes of this study primipara is defined as women whose pregnancy has resulted in a stillbirth or live birth after 28 weeks of gestation. Multiparous women were women with more than one pregnancy which resulted in either a stillbirth or live birth following 28 weeks.

The registry is continuously evaluated by the National Board of Health and the conclusion of the latest extensive validation in 2002 was that quality of data is high [24]. In the present study, we used the same limits for data acceptance as in the Swedish Perinatal Quality Registry, i.e. records of infants with a birth weight $(\mathrm{BW})<200$ or $>9998 \mathrm{~g}$ and a birth length $(\mathrm{BL})<15$ or $>65 \mathrm{~cm}$ were excluded. Applying these limits, no records were excluded due to BW but 90 records were excluded due to extreme values on BL. We also excluded records with extreme values on maternal age $(<13$ or $>54$ years $)$, weight $(<40$ and $>200 \mathrm{~kg})$ and height $(<120$ and $>200 \mathrm{~cm})$.

Fetal sex and gestational age adjusted reference percentiles for birth weight were calculated based on data from singleton infants, without major malformations and born between $28-43$ weeks in the same time period (1998-2007) to mothers without diabetes, $n=874620$.

\section{Study cohort}

The study was based on national data and included all singleton pregnancies to women with and without T1DM. The study cohort comprised 4092 singleton pregnancies to women with T1DM and 905 565 singleton pregnancies to women without a diagnosis of diabetes and referred to as the general population.

\section{Outcomes}

Maternal outcomes of interest include hypertensive disorders in pregnancy; preeclampsia, defined as a resting blood pressure (BP) of $\geq 140 / 90$ and proteinuria $\geq 0.3 \mathrm{~g} / 24 \mathrm{~h}$ or $\geq 2+$ on a dipstick from the $20^{\text {th }}$ week of gestation and gestational hypertension (GH) defined as BP $\geq 140 / 90$ after the $20^{\text {th }}$ week of gestation. Fetal and neonatal outcomes include major malformations (malformation that is potentially life threatening or leading to a major cosmetic defect if not surgically corrected); large for gestational age (LGA): birth weight $>90^{\text {th }}$ percentile for sex and gestational age; small for gestational age (SGA): birth weight $<10^{\text {th }}$ percentile for sex and gestational age; preterm delivery (birth $<37$ weeks of gestation); perinatal death (PMR: stillbirth after 28 completed weeks of gestation or neonatal death before 7 days of life) and late neonatal death, defined as death after the first week but within the first 28 days of life.

\section{Statistical analysis}

Continuous data were summarized by the median and interquartile range and univariate comparison between groups were performed using the Mann Whitney $\mathrm{U}$ test. Comparison of dichotomous data was performed using the Chi-squared test and Fischer's exact test as appropriate. Logistic regression analysis was used to analyze the association between parity and the risk of adverse pregnancy outcome. Primiparous women served as the reference category in the logistic regression models with comparison of pregnancy outcomes in multiparous women in both groups with and without T1DM. Potential confounders included in the multivariate analysis were maternal age, smoking, cohabitation, maternal pre-pregnancy body mass index (BMI), country of birth (Nordic/non-Nordic) and mode of delivery. The estimates were also adjusted for previous adverse pregnancy outcome defined as either of the following diagnoses: previous preeclampsia or gestational hypertension, perinatal death, preterm delivery or major fetal malformation. In the multivariate regression models missing indicator variables for maternal age and BMI were used. Interaction between TIDM and parity was tested using the likelihood ratio test comparing adjusted models with and without an interaction term. A p-value of $<0.05$ indicates a significant interaction between parity and T1DM for outcome of interest. Cluster analysis was performed to account for repeated pregnancies. The study was based on prospectively collected data and including the whole population, limiting the risk of information and selection bias. All analyses were performed using statistical software STATA 10.

\section{Results}

In the study cohort, $45 \%(\mathrm{n}=1851)$ of women with T1DM and $44 \%$ $(n=404338)$ of women in the general population were primiparous ( $p$ $<0.05$ ). The parity distribution was almost identical in women with and without T1DM with approximately $45 \%$ of women with one birth, $36 \%$ with two births, $14 \%$ with three births and $5 \%$ with four or more births (data not shown). Independent of type 1 diabetes status, compared to multiparous women primiparous women were more likely younger, had lower BMI, were smokers and of Nordic origin. They were also less likely to live with their partner compared to multiparous women (Table 1). For both women with and without T1DM, preterm deliveries were significantly more common in primiparous women. The median birth weight was higher in neonates of multiparous women. Instrumental delivery and cesarean section were significantly more common in primiparous women in both groups.

The frequency of all outcomes was significantly higher in women with T1DM irrespective of parity status (p-value $<0.01$, Table 2 ). In both the general population and within the T1DM cohort, rates of most outcomes were higher in primaparous than in multiparous women. In offspring of mothers with T1DM, the frequency of major malformations was higher in mutiparous as compared with primaparous women (Table 2). In both women with and without T1DM, the frequency of LGA infants was significantly higher in multiparous women.

In contrast to the findings in the general population, the odds of gestational hypertension and major malformations did not differ with parity in women with T1DM in either the crude or the adjusted models (Tables 3 and 4). After adjusting for potential confounders, the odds of preterm delivery did not differ significantly between primiparous and muliparous women with T1DM (Table 4). Adding the covariate previous adverse outcome to the multivariate model did not significantly change the estimates in women with or without T1DM. The adjusted odds ratio for LGA was similar in multiparous women with and without T1DM (Table 4). There was a significant interaction between T1DM and parity for preterm delivery but not for any of the other outcomes. The difference in risk associated with parity was greater amongst women without Type 1 diabetes compared to women with Type 1 diabetes. Cluster analysis, to account for the effect of women contributing more than one pregnancy to the cohort, did not significantly change the estimates (data not shown).

\section{Discussion}

In this large population-based study we found a similar pattern of 
Citation: Persson M, Pasupathy D (2015) Effect of Parity on Pregnancy Outcome in Women with Type-1 Diabetes - A Swedish Population-based Study. J Diabetes Metab 6: 534. doi:10.4172/2155-6156.1000534

Page 3 of 5

\begin{tabular}{|c|c|c|c|c|}
\hline & \multicolumn{2}{|l|}{ T1DM, N= 4092} & \multicolumn{2}{|c|}{ Reference group, N=905 565} \\
\hline & Primipara & Multipara & Primipara & Multipara \\
\hline \multicolumn{5}{|l|}{ Maternal characteristics } \\
\hline Age, years* & $28(25-32)$ & $32(28-35)$ & $28(25-31)$ & $31(28-35)$ \\
\hline Nordic & $1746(94.3)$ & $2028(90.5)$ & $340758(84.7)$ & $415794(82.6)$ \\
\hline Living with partner & $1563(84.4)$ & $1968(87.8)$ & $350203(87.0)$ & $455007(90.4)$ \\
\hline Smoking in early pregnancy & $315(17.0)$ & $341(15.2)$ & 76297 (19.0) & $70023(13.9)$ \\
\hline Body mass index ${ }^{*}$ & $24.7(22.7-27.7)$ & $25.5(23.2-29.3)$ & $23.1(21.2-25.8)$ & $23.9(21.7-26.8)$ \\
\hline Height, $\mathrm{cm}^{*}$ & $167(162-171)$ & $166(162-170)$ & $167(162-171)$ & $167(162-170)$ \\
\hline \multicolumn{5}{|l|}{ Infant characteristics } \\
\hline Male & $926(50.0)$ & $1162(51.9)$ & $207225(51.5)$ & $258815(51.4)$ \\
\hline Gestational age, weeks* & $38(37-39)$ & $38(37-39)$ & $40(39-41)$ & $40(39-40)$ \\
\hline Preterm birth (<37 weeks) & $450(24.3)$ & $466(20.8)$ & $24653(6.1)$ & $20087(4.0)$ \\
\hline Birth weight* & $\begin{array}{c}3665 \\
(3185-4122)\end{array}$ & $\begin{array}{c}3900 \\
(3450-4340)\end{array}$ & $\begin{array}{c}3475 \\
(3145-3810)\end{array}$ & $\begin{array}{c}3640 \\
(3305-3980)\end{array}$ \\
\hline Birth length* & $50(49-52)$ & $51(49-52)$ & $50(49-52)$ & $51(49-52)$ \\
\hline \multicolumn{5}{|l|}{ Mode of delivery } \\
\hline Cesarean section & $1025(55.4)$ & $1063(47.5)$ & $70610(17.6)$ & $68311(13.6)$ \\
\hline Ventouse /forceps & $325(17.6)$ & $108(4.8)$ & $54396(13.5)$ & $14106(2.8)$ \\
\hline
\end{tabular}

${ }^{*}$ Comparisons were made using the Mann Whitney $U$ test. *Numbers are medians and interquartile ranges.

Table 1: Maternal and infant characteristics and mode of delivery, stratified by maternal diabetes.

\begin{tabular}{|c|c|c|c|c|c|c|}
\hline & \multicolumn{3}{|c|}{ T1DM N=4092 } & \multicolumn{3}{|c|}{ Reference group N=905 565} \\
\hline & $\begin{array}{l}\text { Primipara } \\
N=1851\end{array}$ & $\begin{array}{l}\text { Multipara } \\
\mathrm{N}=2241\end{array}$ & P-value & $\begin{array}{l}\text { Primipara } \\
N=402338\end{array}$ & $\begin{array}{c}\text { Multipara } \\
\mathrm{N}=503227\end{array}$ & P-value \\
\hline Preeclampsia & $396(21.4)$ & $223(10.0)$ & $<0.001$ & $16751(4.2)$ & $8201(1.6)$ & $<0.001$ \\
\hline Gestational hypertension & $39(2.1)$ & $34(1.5)$ & 0.156 & $4492(1.1)$ & $3307(0.7)$ & $<0.001$ \\
\hline Major malformation & $73(3.9)$ & $96(4.3)$ & 0.586 & $7402(1.8)$ & $8635(1.7)$ & $<0.001$ \\
\hline Perinatal mortality & $31(1.7)$ & $16(0.7)$ & 0.004 & $2319(0.6)$ & $1628(0.3)$ & $<0.001$ \\
\hline Preterm delivery & $450(24.3)$ & $466(20.8)$ & 0.007 & $24653(6.9)$ & $20087(4.0)$ & $<0.001$ \\
\hline SGA & $84(4.5)$ & $54(2.4)$ & $<0.001$ & $55248(13.7)$ & $37262(7.4)$ & $<0.001$ \\
\hline LGA & $782(42.3)$ & $1231(54.9)$ & $<0.001$ & $29570(7.4)$ & $66689(13.3)$ & $<0.001$ \\
\hline Late neonatal death & $3(0.2)$ & $5(0.2)$ & 0.736 & $171(0.04)$ & $199(0.04)$ & 0.489 \\
\hline
\end{tabular}

*Preterm birth: birth before 37 completed weeks. SGA/LGA: small/large for gestational age. Late neonatal death: death after the first week but within the first 28 days of life. ${ }^{*}$ Comparisons were made using the Chi-square test and the Fischer's exact test as appropriate.

Table 2: Outcome variables in numbers and percentages (\%).

\begin{tabular}{|c|c|c|c|c|c|c|}
\hline & \multicolumn{2}{|c|}{ T1DM } & \multirow[b]{2}{*}{ P-value } & \multicolumn{2}{|c|}{ Reference group } & \multirow[b]{2}{*}{ P-value } \\
\hline & Primipara & Multipara & & Primipara & Multipara & \\
\hline Preeclampsia & 1.0 & $0.41(0.34-0.48)$ & $<0.001$ & 1.0 & $0.38(0.37-0.39)$ & $<0.001$ \\
\hline Gestational hypertension & 1.0 & $0.72(0.45-1.14)$ & 0.16 & 1.0 & $0.59(0.56-0.61)$ & $<0.001$ \\
\hline Major malformation & 1.0 & $1.09(0.80-1.49)$ & 0.59 & 1.0 & $0.93(0.90-0.96)$ & $<0.001$ \\
\hline Perinatal mortality & 1.0 & $0.42(0.23-0.77)$ & 0.005 & 1.0 & $0.56(0.53-0.60)$ & $<0.001$ \\
\hline Preterm birth & 1.0 & $0.82(0.71-0.95)$ & 0.007 & 1.0 & $0.64(0.62-0.65)$ & $<0.001$ \\
\hline SGA & 1.0 & $0.52(0.37-0.74)$ & $<0.001$ & 1.0 & $0.50(0.50-0.51)$ & $<0.001$ \\
\hline LGA & 1.0 & $1.67(1.47-1.89)$ & $<0.001$ & 1.0 & $1.93(1.90-1.95)$ & $<0.001$ \\
\hline Late neonatal death & 1.0 & $1.38(0.33-5.77)$ & 0.66 & 1.0 & $0.93(0.76-1.14)$ & 0.49 \\
\hline
\end{tabular}

*Preterm birth: birth before 37 completed weeks. SGA/LGA: small/large for gestational age. Late neonatal death: death after the first week but within the first 28 days of life.

Table 3: Crude odds ratios with confidence intervals, stratified for parity and diabetes status.

reduced risks of adverse pregnancy outcome with multiparity in both pregnancies with and without maternal T1DM. However, within the T1DM cohort the risk reduction for preterm birth and gestational hypertension with multiparity was less than in the general population. Multiparty was associated with a slightly increased risk of major malformations in offspring of mothers with T1DM, as opposed to the findings in the general population. In both pregnancies with and without maternal diabetes, multiparity was associated with increased risk for delivery of large for gestational age infants.

The observation of increased risks in primaparous women without diabetes is in keeping with a large number of previous reports [5-12]. It has been proposed that the increased risk of adverse outcome associated with primiparity in women without diabetes can partly be explained by a higher prevalence of maternal risk factors such as young age, low 


\begin{tabular}{|c|c|c|c|c|}
\hline & & \multicolumn{2}{|c|}{ Reference } \\
\hline & Primipara & Multipara & Primipara & Multipara \\
\hline Preeclampsia & 1.0 & $0.35(0.28-0.43)$ & 1.0 & $0.36(0.35-0.37)$ \\
\hline Gestational hypertension & 1.0 & $0.63(0.37-1.05)$ & 1.0 & $0.49(0.46-0.52)$ \\
\hline Major malformation & 1.0 & $1.25(0.88-1.77)$ & 1.0 & $0.95(0.92-0.99)$ \\
\hline Perinatal mortality & 1.0 & $0.22(0.10-0.51)$ & 1.0 & $0.44(0.41-0.48)$ \\
\hline Preterm birth & 1.0 & $0.83(0.70-1.00)$ & 1.0 & $0.66(0.65-0.68)$ \\
\hline SGA & 1.0 & $0.44(0.29-0.68)$ & 1.0 & 0.39 \\
\hline LGA & 1.0 & $1.96(1.71-2.25)$ & 1.0 & $0.52(0.51-0.52)$ \\
\hline Late neonatal death & 1.0 & $0.56(0.12-2.60)$ & 1.0 & $1.92(1.89-1.95)$ \\
\hline
\end{tabular}

*Preterm birth : birth before 37 completed weeks. SGA/LGA: small/large for gestational age. Late neonatal death: death after the first week but within the first 28 days of life. All models include adjustment for mother's country of birth, smoking before pregnancy, cohabit, maternal age, pre-pregnancy BMI, mode of delivery and previous adverse outcome. Interaction term: interaction between parity and T1DM, a p-value of $<0.05$ indicates a significant interaction.

Table 4: Adjusted odds ratios, stratified for parity and diabetes status.

pre-pregnancy BMI, insufficient pregnancy weight gain, hypertensive disorders of pregnancy, poor education, non-cohabiting, smoking and socioeconomic stress [13-15]. However, we found that the odds of obstetric complications, neonatal morbidity and perinatal mortality remained significantly increased in primiparous women without diabetes also after adjusting for several of these maternal confounders.

This study is to our knowledge the first national study to explore the risk of pregnancy complications in relation to parity in women with T1DM. The population-based study design and prospectively collected data limit the risk of selection and information bias. We were also able to adjust for several important confounders such as maternal age, smoking, ethnicity and pre pregnancy BMI. In Sweden health care is free at the point of care which includes equipment for glucose monitoring and insulin administration such as insulin and test strips. Pregnancy care of women with T1DM is uniform in the country and pregnancy outcomes do not differ with hospital size or geographical area $[1]$.

Our study had some limitations. Even though this study was based on national data of all singleton T1DM pregnancies in Sweden over a period of eleven years, the statistical power to detect differences was lower within the T1DM cohort compared with the much larger reference group and may also limit the possibility to detect significant interaction. Therefore, the results must be interpreted with some caution. In addition, we did not have data on duration of diabetes or the presence of diabetes microangiopathy and residual confounding cannot be excluded. Women with T1DM are regularly informed about the potential problems associated with pregnancy and about the importance of family planning. Thus, this group of women should be prepared for the risk of adverse pregnancy outcome. It might be that the risk of complications is outweighed by the strong desire for a child which may contribute to the diverging results regarding the risk for major malformations between women with and without type 1 diabetes. This however, cannot be tested in our dataset.

In Sweden pre-pregnancy counseling is readily provided through adolescence diabetes clinics and is regularly reinforced. The single most important measure in order to reduce the risk of complications in T1DM pregnancies is striving towards a tight glycemic control. Maternal hyperglycemia is a well-known risk factor for both preterm delivery and major malformations $[17,18,20]$. The risk reductions with multiparity for preterm delivery and major malformations were less pronounced in women with T1DM. Thus it may well be that the level of achieved degree of glycemic control was similar in primiparous and multiparous women. In addition multiparous women are likely to have longer duration of diabetes with physiological sequelae of the disease reflected in the higher risk of some of the adverse outcomes in the multiparous population. These hypotheses cannot be tested for in the present study as the MBR does not collect data on level of glycemic control and severity of co-morbidities related to diabetes in both nulliparous and multiparous women. However, data from other studies indicate that the level of control possible is similar between pregnancies and striving towards normoglycemia must be balanced against the risk of maternal hypoglycemia that may vary between patients $[24,25]$.

Multiparous women had significantly lower risks for preeclampsia in both pregnancies with and without maternal diabetes. It has been suggested that the increased risk of preeclampsia in primiparous women may be attributed to immunological factors unique to the first pregnancy [15].

All women with T1DM are offered a detailed ultrasound examination between the $18^{\text {th }}$ and $20^{\text {th }}$ week of gestation with the primary aim to detect malformations. Unfortunately, the MBR does not collect information on induced abortions or early fetal losses (before $22^{\text {nd }}$ week of gestation). However, as the risk of malformations was independent of parity it is unlikely that an increased rate of induced abortions in multiparous women would bias our findings. One possible contributing factor to the higher recorded risk of complications in nulliparous women could be the higher prevalence of fertility problems in this group. The lack of data on early fetal losses limits our possibility to take this factor into account in the analyses.

High BMI is a well-known risk factor for both maternal and fetal complications, including LGA [25]. Results from the HAPO study clearly demonstrate that maternal BMI is associated with increased risk for LGA, independent of maternal glycaemia at oral glucose test [26]. Mean maternal BMI was higher in multiparous than primaparous women and may have contributed to the increased risk for LGA.

In conclusion, this study demonstrates that multiparity is associated with reduced risks for adverse pregnancy outcome in both pregnancies with and without T1DM with exception of an increased risk for large for date infants. In pregnancies with T1DM, the risk reduction for major malformations and preterm birth is less pronounced than in the general population. We believe that the findings are applicable to other similar populations. Several studies have reported favorable results of pre-pregnancy counseling on the occurrence of major malformations and may also decrease the risk of preterm delivery [27]. In order to further improve pregnancy outcome in women with type 1 diabetes it would be of importance to encourage attendance to pre-pregnancy clinics. 
Citation: Persson M, Pasupathy D (2015) Effect of Parity on Pregnancy Outcome in Women with Type-1 Diabetes - A Swedish Population-based Study. J Diabetes Metab 6: 534. doi:10.4172/2155-6156.1000534

Page 5 of 5

\section{Funding}

This study was supported by unrestricted research grants from the Stockholm City Council. DP was funded by the National Institute of Health Research, UK and Tommy's Charity, UK

\section{Acknowledgement}

The authors are very grateful to Ulf Hanson, associate professor at the Department of Obstetrics and Gynaecology University of Uppsala, for kindly sharing his data with us. The data for this analysis was obtained from the Swedish Medical Birth Registry which was provided by the National Board of Health and Welfare.

\section{References}

1. Persson M, Norman M, Hanson U (2009) Obstetric and perinatal outcomes in type 1 diabetic pregnancies- a large population-based study. Diabetes Care 32: 2005-2009.

2. Vaarasmaki M, Gissler M, Hartikainen AL (2001) A uniform regimen enables decentralized care of diabetic pregnancies. Diab Med 18: 871-876.

3. EversIM, deValkHW,VisserGH(2004)Riskofcomplicationsofpregnancyinwomen withtype 1 diabetes:nationwideprospectivestudyintheNetherlands.BMJ328:915 Jensen DM, Damm P, Moelsted-Pedersen L, Ovesen P, Westergaard JG, et al. (2004) Outcomes in type 1 diabetic pregnancies: a nationwide, populationbased study. Diabetes Care 27: 2819-2823.

4. Duckitt K, Harrington D (2005) Risk factors for pre-eclampsia at antenata booking: systematic review of controlled studies. BMJ 12: 330-565.

5. Raymond EG, Cnattingius S, Kiely JL (1994) Effects of maternal age, parity, and smoking on the risk of stillbirth. Br J Obstet Gynaecol 101: 301-306.

6. Bai J, Wong FW, Bauman A, Moshin M (2002) Parity and pregnancy outcome. Am J Obstet Gynecol 186: 274-278.

7. Ananth CV, Basso O (2010) Impact of Pregnancy-Induced Hypertension on Stillbirth and Neonatal Mortality in First and Higher Order Births:A populationBased Study. Epidemiology 21: 118-123.

8. Ananth CV, Peltier MR, Getahun, Kirby RS, Vintzileos AM (2007) Primaparity: An "intermediate" risk group for spontaneous and medically indicated preterm birth. J Matern Fetal Neonatal Med 20: 605-611.

9. Shoham-Vardi I, Lieberman JR, Kopernik G (1994) The association of primaparity with intrauterine growth retardation. Eur J Obstet Gynecol Reprod Biol 53: 95-101.

10. Zhang X, Mumford SL, Cnattingius S, Schisterman EF, Kramer MS (2010) Reduced birthweight in short and primaparous mothers:physiological or pathological? BJOG 117: 1248-1254.

11. Kramer MS, Olivier M, McLean FH, Dougherty GE, Willis DM, et al. (1990) Determinants of fetal growth and body proportionality. Pediatrics $86: 18-26$.
12. Lee KS, Ferguson RM, Corpuz M, Gartner LM (1998) Maternal age and incidence of low birth weight at term: a population study. Am J Obstet Gynecol 158: 84-89.

13. Wen SW, Goldenberg RL, Cutter GR, Hoffman HJ, Cliver SP (1990) Intrauterine growth retardation and preterm delivery:prenatal risk factors in an indigent population. Am J Obstet Gynecol 162: 213-218.

14. Sibai B, Dekker G, Kupferminc M (2005) Pre-eclampsia. Lancet 365: 785-799.

15. Miranda ML, Edwards SE, Myers ER (2011) Adverse Birth Outcomes Among Nulliparous vs. Multiparous Women. Public Health Reports 126: 797-805.

16. Greene MF et al. (1989) First trimester hemoglobin A1 and risk for major malformation and spontaneous abortion in diabetic pregnancy. Teratology 39 : 225-231.

17. Ylinen K, Aula P, Stenman UH, Kesäniemi-Kuokkanen et al. (1984) Risk of minor and major fetal malformations in diabetics with high haemoglobin A1c values in early pregnany. Br Med J(Clin Res Ed) 289: 345-346.

18. Lauenborg J, Mathiesen E, Ovesen P, Westergaard JG, Ekbom P, et al. (2003) Audit on stillbirths in women with pregestational type 1 diabetes. Diabetes Care 26: $1385-1389$.

19. Ekbom $P$ et al. (2008) Elevated third-trimester haemoglobin A 1c predicts preterm delivery in type 1 diabetes. J Diabetes Complications 22: 297-302.

20. Persson M, Pasupathy D, Hanson U, Norman M (2012) Pre-pregnancy body mass index and the risk of adverse outcome in type 1 diabetic pregnancies: a population-based cohort study. BMJ Open 2: e000601.

21. Ekbom P, Damm P, Feldt-Rasmussen B, Feldt-Rasmussen U, Mølvig J, et al. (2001) Pregnancy outcome in type 1 diabetic women with microalbuminuria. Diabetes Care 24: 1739-1744.

22. The Swedish Centre for Epidemiology (2003) The Swedish Medical Birth Register. A summary of content and quality.

23. Nielsen LR, Pedersen-Bjergaard $U$, Thorsteinsson B, Johansen $M$, Damm $P$ et al. (2008) Hypoglycemia in pregnant women with type 1 diabetes:predictors and role of metabolic control. Diabetes Care 31: 9-14.

24. Persson B (1974) Ciba Foundation Symposium, Elsevier 27: 247-274.

25. HAPO study Cooperative Research Group (2010) Hyperglycaemia and Adverse Pregnancy Outcome (HAPO) Study: associations with maternal body mass index. BJOG 117: 575-584.

26. Kitzmiller JL, Buchanan TA, Kjos S, Combs CA, Ratner RE (1996) Preconception care of diabetes, congenital malformations, and spontaneous abortion. Diabetes Care 19: 514-541.

27. Wahabi HA, Alzeidan RA, Esmail SA (2012) Pre-pregnancy care of women with diabetes mellitus:a systematic review and meta-analysis. BMC Public Health 12: 792 . 